\title{
Time To Live (TTL) Impact on the Performance of STAR Protocol in MANETs
}

\author{
C P V N J Mohan Rao
}

Professor, Avanthi Institute of Engineering and Technology, Makavarapalem,Narsipatnam, India

mohanrao_c@yahoo.com

S. Pallamsetty

Professor, Department of CS\&SE, Andhra University, Visakhapatnam, India

drspsetty@gmail.com

P V G D Prasad Reddy

Professor, Department of CS\&SE, Andhra University, Visakhapatnam, India

prasadreddy.vizag@gmail.com

\begin{abstract}
Mobile Ad hoc Network (MANET) is a developing area in the existing mobile environment. Its standards are defined by IETF. MANETs consists of several characteristics like dynamic topology, easy deployment and robustness which make them as a striking topic for the research community. Routing, enhancing the QoS is a challenging issue in MANETs. In this paper, one of the best proactive routing protocols ie Source Tree Adaptive Routing (STAR) protocol has been chosen. The selection of the stable configurable values in protocol will affect QoS performance. In MANETs, Time to Live (TTL) is a constant value and it has to be configured with network size accordingly. The impact of TTL value on the performance of STAR routing protocol has been analyzed. Simulation results suggest that the impact of TTL shows significant change on the performance of STAR at all network sizes with lower mobility. This paper shows that static values like TTL are not suitable for dynamic environment in protocol configuration and suggests that the TTL value should be a varied with the networksize and mobility speed accordingly to achieve better performance.
\end{abstract}

Keywords: MANET, IETF, TTL, STAR, QoS

\section{INTRODUCTION}

Mobile Ad hoc Network is a kind of ad hoc network and consists of collection of wireless mobile nodes forming a temporary network without the help of centralized administration. These networks are selfconfigured, self -organized and self-handled [1]. Each node in the network acts as both transceiver as well as router. All the nodes move frequently in the network region. MANETs have several characteristics like dynamic topology, Bandwidth utilization, ease of deployment, routing, high mobility of nodes make them an attractive topic for the research community [2]. Routing and QoS are the two major issues in MANETs out of several challenging issues.

Routing is the selection of best route from source to destination in a network for the transfer of packets. It is done in multi-hop manner with the help of intermediate nodes within the internetwork for the transfer of information [3]. Routing protocols for ad-hoc network can be categorized in to three types- Proactive, Reactive and Hybrid routing protocols. Proactive routing is also called Table driven routing whereas Reactive routing is called On-demand \& dynamic routing [4,5]. STAR is one of the popular proactive routing protocols [6].

\section{STAR ROUTING PROTOCOL}

In STAR, each router reports to its neighbors the features of each link it utilizes to access a destination. Router's source tree is called the set of connections that a router utilizes in its preferred route to destinations. A router understands its neighboring connections and the source trees recorded by its neighbours; a complete topology graph is the aggregation of the neighboring connections of a router and the source trees recorded by its neighbours. The connections must be neighboring connections or connections recorded by at least one neighbour in the source tree and topology graph. The router uses the topology graph to generate its own source tree. Each router derives a routing table specifying the successor to each destination by running a local routeselection algorithm on itssource tree. The ORA or LORA approach can be used to update routing information depending on the bandwidth available on an adhoc network. Both are supported by Star.

In ORA, when source tree changes updates are sent. Where as in LORA, a router running STAR only sends updates to its neighbors on its source tree if it loses all paths to one or more destinations, if it detects a new destination or if it determines that local changes to its source tree could potentially create long-term routing loops [6]. 


\section{RELATED WORK}

Researchers evaluated the efficiency of different proactive routing protocols in mobile adhoc networks. We summarize the present status of the representative samples. Most of them used QoS metrics, viz., throughput, jitter and end-to-end delay to asses the performance. They attempted primarily on analyzing the effect of Time to Live (TTL) on the performance of the STAR routing protocol. B.Prasad et al., evaluated TTL's effect on Energy Aware Routing Protocol in MANET and emphasized its important impact [7]. Madhusudan et. al., proposed robust throughput and packet transfer capacity measures to characterize the ability of a mobile ad hoc wireless network to provide highly survivable transport of data flows in real time communication. They opined that a network system that is designed to yield a high throughput rate does not necessarily provide its users with a high measure of robust service [8]. Raju et. al., analyzed NNT's effect on AODV and designed a new FBNTTAODV protocol that delivers stronger output than AODV [9]. Edenhofer et. al., has published a paper titled Towards a Rigorous Analysis of AODVv2 (DYMO) by making a detailed comparision between AODV and AODVv2 [10]. Yogesh Chaba et. al, compared the performance of reactive protocols and found that all reactive routing protocols have flat routing structure except in case of CBRP, which uses the hierarchical structure. Further, network scalability depends on the level of traffic and the levels of multi hoping which may be up to few hundred nodes but pointto point may scale higher [11]. Node traversal time (NTT) is a conservative assessment of the average one hop traversal time for packets which includes queuing delays, interrupt processing times and transfer times. IFIP International Conference on Network and Parallel Computing proceedings edited by Jian Cao et. al., set out the importance of NTT and given the procedure for its calculation in a complex network. [12].

\section{METHODOLOGY}

According to the IETF draft, the timing parameters for STAR should be configurable administratively. Ideally, the STAR parameters should be modified with either experimentally defined values or dynamic adoptation with frequent topology changes. As per the characteristics of a network routing protocol TTL plays a vital role. If TTL is too low, connection cannot be established and if it is too high energy consumption increases, which in turn affects the network life time. For example, in Qualnet simulator, TTL's default value for the STAR routing protocol is 6 . but it does not show how appropriate this value is for different network sizes. TTL is one of the key parameters for the protocol. In a dynamic topology, due to differing mobility and different network sizes, we are not supposed to take fixed values. This paper assesses STAR routing protocol quality by varying TTL values from 1 to 6 under different network sizes and mobility speeds. Initially, STAR's output was checked with a stable default value i.e. TTL as 6. Later, the default TTL value was changed from 1 to 5 and the experimentation is repeated.

\subsection{Simulation Environment}

QualNet is a network modeling software that is fast, scalable and high-fidelity. This is the GloMoSim open source simulator scalable version. It allows new network technologies to be developed very efficiently and costeffectively. Qualnet's key advantages are speed and scalability. QualNet can support network emulation, hardware-in - the-loop and human-in - the-loop simulations in real-time with faster simulation speed. QualNet provides support for thousands of nodes. Parallel computing architectures can also be used to support more network nodes and faster simulation. Speed and scalability with QualNet are not mutually exclusive [13].

\subsection{Simulator Setting and Execution}

QualNet simulator is used to test the STAR routing protocol with LORA simulation scenarios. Table 1 displays the parameters and values taken for different inputs to create the scenarios.

Table 1: Simulation Scenario Parameters

\begin{tabular}{|l|l|}
\hline Routing Protocol & STAR \\
\hline Simulation Time & $300 \mathrm{~s}$ \\
\hline Area (sq.m) & $1000 \times 1000$ \\
\hline Propagation Model & Two Ray \\
\hline Traffic & CBR \\
\hline Packet Size & 512 bytes \\
\hline Nodes & $20,40,60,100$ \\
\hline Antenna Type & Omni directional \\
\hline Transmission range & $250 \mathrm{~m}$ \\
\hline Receiver range & $250 \mathrm{~m}$ \\
\hline Pause time & 0 sec \\
\hline Min \&Max speed & $1 \mathrm{~m} / \mathrm{s}$ to $20 \mathrm{~m} / \mathrm{s}$ \\
\hline $\begin{array}{l}\text { Maximum number of buffered } \\
\text { packets }\end{array}$ & 100 \\
\hline Mobility Model & Random Waypoint \\
\hline TTL & $2,3,4,5,6$ \\
\hline
\end{tabular}

\section{RESULTS AND DISCUSSIONS}

The analysis of routing protocols uses different performance metrics. In terms of QoS, they reflect different characteristics of overall network efficiency. In this paper, we defined different metrics used in STAR quality evaluation with different TTL values. The measures used are Throughput, Average End-to-End Delay, Average Jitter. 
Throughput: It is the average rate of packets transmitted through a channel of communication. The ratio of the total amount of data entering a receiver from a sender to the amount of time it takes for the receiver to retrieve the last packet is referred to as the thrughput. It is expressed either in bits per second or in packets per second. It is always desirable to have the throughput of a network to be as high as possible. Figure 1 shows the results of the throughput with different TTL values.

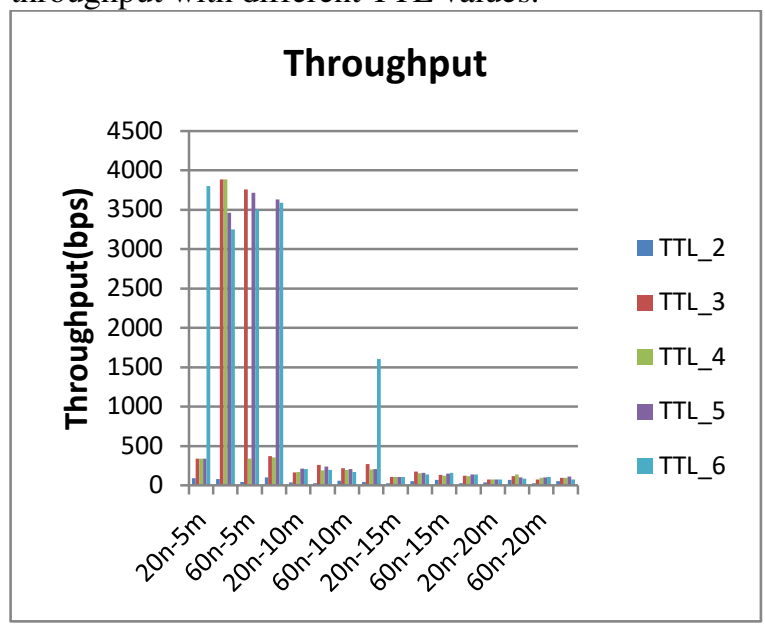

Figure 1 Variation of Throughput with TTL

From the experimental results shown in the figure 1, it is observed that, change in the value of TTL has more impact on the throughput in small and medium networks with lower mobility speed $(5 \mathrm{~m} / \mathrm{s})$.

End-to-End Delay: The end-to-end delay of the packet is the average time it takes for packets to traverse the network. This is the time from the sender's generation of the packet to its reception on the application layer of the destination and is expressed in seconds. It therefore includes all network delays such as buffer queues, transmission time and delays resulting from routing activities and exchanges of MAC control. The delay should be minimal for better performance. Figure 2 displays the results of end-to-end delay for various TTL values.

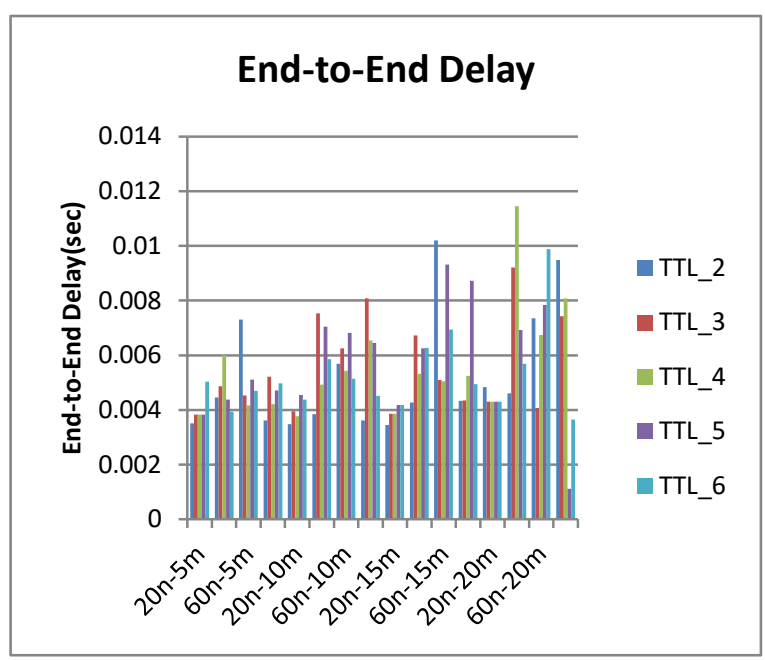

Figure 2 Variation of End-to-End Delay with TTL
From the experimental results, we find that the End-to-End Delay at lower mobility speed $(5 \mathrm{~m} / \mathrm{sec}$ to $10 \mathrm{~m} / \mathrm{sec})$ is minimum for all network sizes. TTL has more impact at lower mobility speeds rather than higher mobility speeds.

Average Jitter: Jitter is the delay variance as measured in the network-wide variability of the packet latency over time. There is no variance or jitter in a network with constant latency. Jitter is a significant QoS factor in network performance evaluation and should be null for real-time networks of communication.

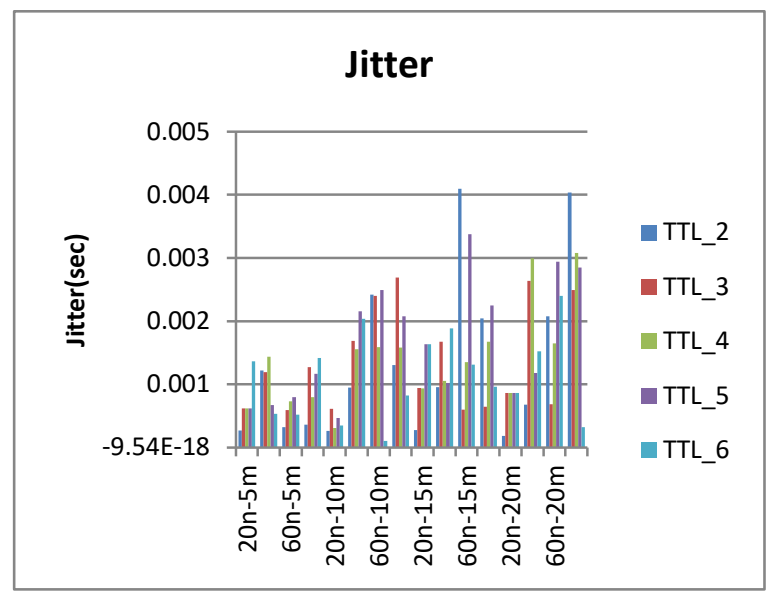

Figure 3 Variation of Jitter with TTL

From the experimental results, we find that for all network sizes with lower mobility speeds $(5 \mathrm{~m} / \mathrm{sec})$ the Average Jitter is minimal.

\section{CONCLUSIONS}

In this paper, one of the best proactive routing protocols viz., Source Tree Adaptive Routing (STAR) protocol with LORA has been chosen for the study. The selection of the steady configurable values in protocol will affect QoS performance. In MANETs, Time to Live (TTL) is a constant value and it has to be configured with network size and mobility speed accordingly. The impact of TTL value on the performance of STAR routing protocol has been analyzed. Simulation results suggest that the impact of TTL shows significant change on the performance of STAR at all network sizes with lower mobility $(5 \mathrm{~m} / \mathrm{sec}$ to $10 \mathrm{~m} / \mathrm{sec}$ ). This study suggests that static values for TTL are not suitable for dynamic environment in protocol configuration and suggests that the TTL value should be varied with the network size and mobility speed for better results in terms of QoS metrics. The work can be further extended by comparing LORA with ORA approach for different network sizes and mobility speeds.

\section{REFERENCES:}

1. Manohar, M. P. and S. P Setty (2018). "Performance Analysis of Reactive Routing Protocols AODV, DYMO, DSR, LAR in MANETs". International Journal on Future Revolutionin Computer Science \& 
Communication Engineering, 4(3), 01-07.

2. VenkataramanaAttada, Pallamshetty S, Performance Evaluation of DSR and LAR Routing Protocols with MAC Layer in MANET's, IJCSMC, Nov2013.

3. S.P Setty and B.Prasad; Comparative Study of Energy Aware QoS for Proactive and Reactive Routing Protocols for Mobile Ad-hoc Networks, International Journal of Computer Applications (0975 8887) Volume 31- No.5, October 2011.

4. S. -J. Lee, M. Gerla and C. -K. Toh, "A Simulation Study of Table-Driven and On-Demand Routing Protocols for Mobile Ad Hoc Networks," IEEE Network, July-August 1999, Vol.13, No. 4, pp.48-54.

5. J. Broch, D. Maltz, D. Johnson, Y.-C. Hu, and J. Jetcheva, "A Performance Comparison of Multihop Wireless Ad Hoc Network Routing Protocols", ACM/IEEE Mobile Computing and Networking, October 1998.

6. Garcia-Luna-Aceves, Spohn, Beyer, IETF MANET Working Group, draft-ietf-manet-star-00.txt, OCT 1999

7. B Prasad, S.P Setty, "Analysing The Impact of TTL Sequence-Based Expanding Ring Search On Energy Aware Routing Protocols for MANETs", International Journal of Engineering Science and Technology (IJEST), Vol. 4 No.05 May 2012.

8. Madhusudan $\mathrm{G}$ and Kumar T N R., "Packet Transfer Rate \& Robust Throughput for Mobile Adhoc Network", Int. J. Advanced Networking and Applications Volume: 08 Issue: 06 Pages: 3242-3245 (2017)

9. Raju and SP Setty; Design and Analysis of Fuzzy based Node Traversal Time AODV for Improving the QoS in Mobile Ad Hoc Networks. International Journal of Computer Applications (0975 - 8887) Volume 58 - Issue 9,November 2012.

10. S. Edenhofer, P. Höfner, Towards a Rigorous Analysis of AODVv2 (DYMO). In N. Foster, A. Gurney (eds.), Rigorous Protocol Engineering (WRiPE'12). IEEE, 2012. doi: 10.1109/ICNP.2012.6459942

11. Yogesh Chaba, Manish Joon, Yudhvir Singh and Anshul, "Analysis of Reactive Routing Protocols for Mobile Ad-Hoc Networks", Int. J. Advanced Networking and Applications Volume: 01, Issue: 02, Pages: 111-115 (2009)

12. Text book proceedings on Network and Parallel Computing: IFIP International Conference, NPC 2008, edited by JianCao, MingluLi, Min-You Wu, JinjunChen.
13. QualNet Network Simulator, Available: http://www.scalable-networks.com

\section{Biographies and Photographs}

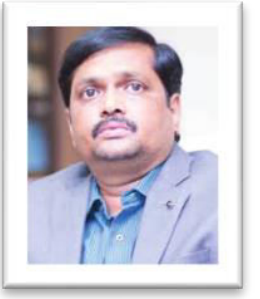

C P V N J Mohan Rao is working as Professor in Department of Computer Science and Engineering at Avanthi Institute of Engineering and Technology, Makavarapalem, Visakhapatnam.. He has 25 years of teaching experience and published more than 18 papers in reputed Journals.

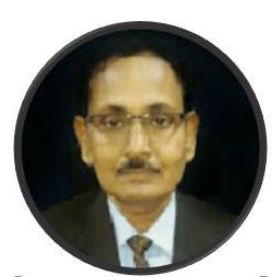

S Pallamsetty is Professor in the Department of Computer Science and Systems Engineering, Andhra University, Visakhapatnam. He has guided $25 \mathrm{PhDs}$ and right now he is guiding ten scholars. He has guided more than 100 M.Tech projects. He received many honors, $\mathrm{He}$ received Gold Medal for securing first rank in M.Sc. He was the He visited Berkley University,U.S.A. He is a Life Member of IETE. He is the Editorial Board Member of various International Resource person for various National and International Conferences and Workshops. Journals like IJCA, IJCIIS, and IJSAT etc.

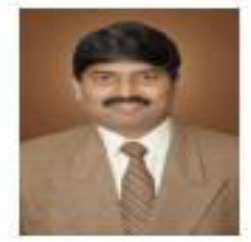

Prasad Reddy, P.V.G.D, was born in Andhra Pradesh. He obtained his M.Tech, in Computer Science \& Technology, and Ph.D, in Computer Engineering. He is presently the BOS, CS \& SE department, Andhra University, Visakhapatnam. His Research areas include Soft Computing, Software Architectures, knowledge Discovery from Databases, Image Processing, Number theory \& Cryptosystems. 